\title{
Constraining the equation of state of the dense matter from X-ray burst oscillations
}

\author{
Romain Artigue* and Didier Barret \\ Université de Toulouse; UPS-OMP; IRAP; Toulouse, France \\ CNRS; IRAP; 9 av. colonel Roche, BP 44346, F-31028 Toulouse cedex 4, France \\ E-mail: romain.artiguedcesr.fr, didier.barretecesr.fr
}

\begin{abstract}
We show that detailed modeling of X-ray burst oscillations can be an extremely powerful probe of the neutron star structure (M, R), and thus the equation of state of ultra-dense matter. $\mathrm{M}$ and $\mathrm{R}$ are encoded in the shape of the oscillations resulting from the spin modulation of the thermonuclear burst flux from the neutron star. We have developed an expanding hot spot model for X-ray burst oscillations, taking into account all relativistic effects affecting photon trajectories from the neutron star surface to the observer. We have simulated light curves assuming different $\mathrm{M}, \mathrm{R}$, hot spot geometry and emission pattern. We show that an improvement by at least a factor of 10 in count statistics (as achieved by LOFT) is required to detect the harmonic content of the signal, with the amplitude of the harmonics directly related to the asymmetry of the waveform.

Using a library of theoretical light curves, we have then fitted the simulated light curves to evaluate the accuracy with which $\mathrm{M}$ and $\mathrm{R}$ could be recovered. We show that $\mathrm{M}$ and $\mathrm{R}$ can be constrained within less than $15 \%$ from one single burst. Allowing several bursts to be co-added, the statistical uncertainty in $\mathrm{M}$ and $\mathrm{R}$ can be further reduced within a few percent, as required to discriminate between for the various equations of state of dense matter.
\end{abstract}

Fast X-ray timing and spectroscopy at extreme count rates: Science with the HTRS on the International $X$-ray Observatory - HTRS 2011,

February 7-11, 2011

Champéry, Switzerland

\footnotetext{
* Speaker.
} 


\section{Introduction}

The ultra-dense neutron star core (which could contain the most exotic form of matter observable in the Universe) is governed by the particle interactions determining the internal pressure $\mathrm{P}$ and density $\rho$. The high-density, low temperature limit is predicted to be marked by the appearance of phase transition to quark-gluon plasma, or pion and kaon condensates, etc. Currently, the properties of the neutron star crust are reasonably well understood, whereas the composition of the neutron star core remains largely unknown. There exist several P- $\rho$ equations, defining each a mass-radius relation (Lindblom (1992) [1]). To discriminate between the proposed equations of state of dense matter, one must obtain simultaneous constraints on $\mathrm{M}$ and $\mathrm{R}$ for a wide sample of neutron stars, of different masses and radii.

Several techniques exist to constrain M and R. The modeling of the (nearly) coherent oscillations during type-I X-ray bursts (Strohmayer (2004) [2]) is a promising one. X-ray bursts are thermonuclear flashes of the accreted matter (H or He) onto the surface (Cumming (2004) [3]) of the neutron star. Type-I X-ray bursts are recurrent phenomenons (hours or days) with a rising phase of 1 or 2 seconds, lasting at least 10 seconds while the surface cools down.

Spectral analysis of the rising part of X-ray burst shows they are likely caused by an expanding blackbody hot spot rotating at the neutron star surface (see Strohmayer et al. (1997) [4]). The spin frequency of neutron stars with burst oscillationsranges between 200 and $600 \mathrm{~Hz}$. The waveform of the oscillations is shaped by relativistic effects, expected in the immediate vicinity of the neutron star. Fitting of the waveform yields simultaneous constraints on M and R. Fortunately, X-ray bursters (in which the neutron star mass grows through accretion) may probe the high mass end of the M-R diagram, where it is easier to discriminate between the different equations of state.

\section{X-ray burst modeling and ray-tracing}

We consider an anisotropic blackbody emission of an expanding hot spot onto the surface of the rotating star. We study the case of a circular and linear expansion. In order to compute the light curve of the rising phase of the burst, we sum the fluxes from discrete surface area elements. Photons propagate in a Schwarzschild metric. The parameters of the model are: the mass, the radius and the spin frequency of the neutron star, the colatitude of the spot center, the initial spot size, the angular growth rate and the orbital inclination of the spot with respect to the observer. We do not include the flux contribution from the non bursting region of the neutron star and from the

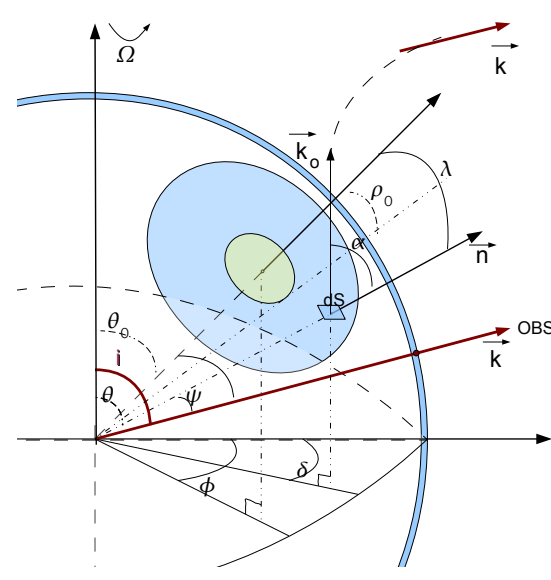

Figure 1: Hot spot geometry. accretion disk (see Fig.1).

We use the approximate analytic formula of the emission derived for the pulse profile produced by small spots on a rapidly rotating neutron star from Poutanen \& Beloborodov (2006) [5]. 


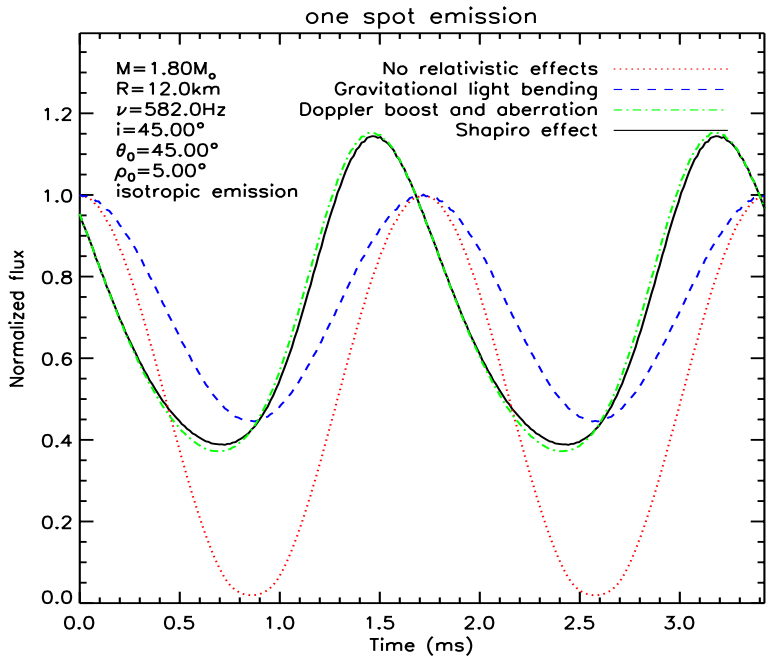

Figure 2: Representation of the relativistic effects on the X-ray burst pulse profile.

On Figure 2 we show the influence of the various processes included in the simulation upon the light curve(from [5]). Light bending (which depends on the compactness $M / R$ ) reduces the relative amplitude of the modulation, because it makes visible the hot spot when it is on the "hidden face" of the neutron star. Doppler effects (related to the rapid rotation) leads to an asymmetrical pulse shape. The Shapiro effect induces a larger delay for photons emitted on the opposite side of the star.

Through the relativistic effects ( $\mathrm{M}$ and R values), the pulse profile becomes asymmetric (see Miller \& Lamb (1998) [6]). This profile must present a significant harmonic component. To detect harmonics in the signal and to obtain constraints on $\mathrm{M}$ and $\mathrm{R}$, the pulse profile must be measured with extremely good statistics.

\section{The statistics achieved by the RXTE/PCA}

Burst oscillations have been detected from 20 systems by the RXTE/PCA (Rossi X-ray Timing Explorer/Proportional Counter Array) instrument (In't Zand [7]), either during the rising or the decaying phase of the bursts. An example is provided in Figure 3, where the statistics achieved on the folded pulse profile is also shown. So far, the harmonic content of the signal has not been detected, preventing meaningful constraints to be derived on the mass and radius (e.g. Nath et al. (2002) [8]).

A systematic analysis of all archival data on $4 \mathrm{U} 1636-536$ is currently underway to test whether the information from individual bursts can be combined to improve on the existing constraints. A set of folded profiles is shown in Figure 4, indicating that the folded profiles do not vary significantly from one burst to the other.

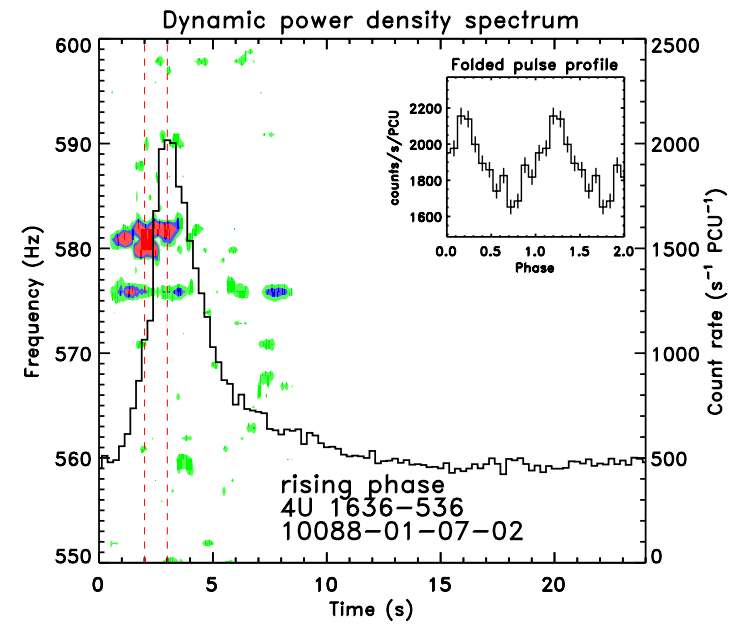

Figure 3: Dynamical power density spectrum of one X-ray burst in 4U 1636-536 observation revealing oscillations during the rising phase of the burst at $581 \mathrm{~Hz}$. 

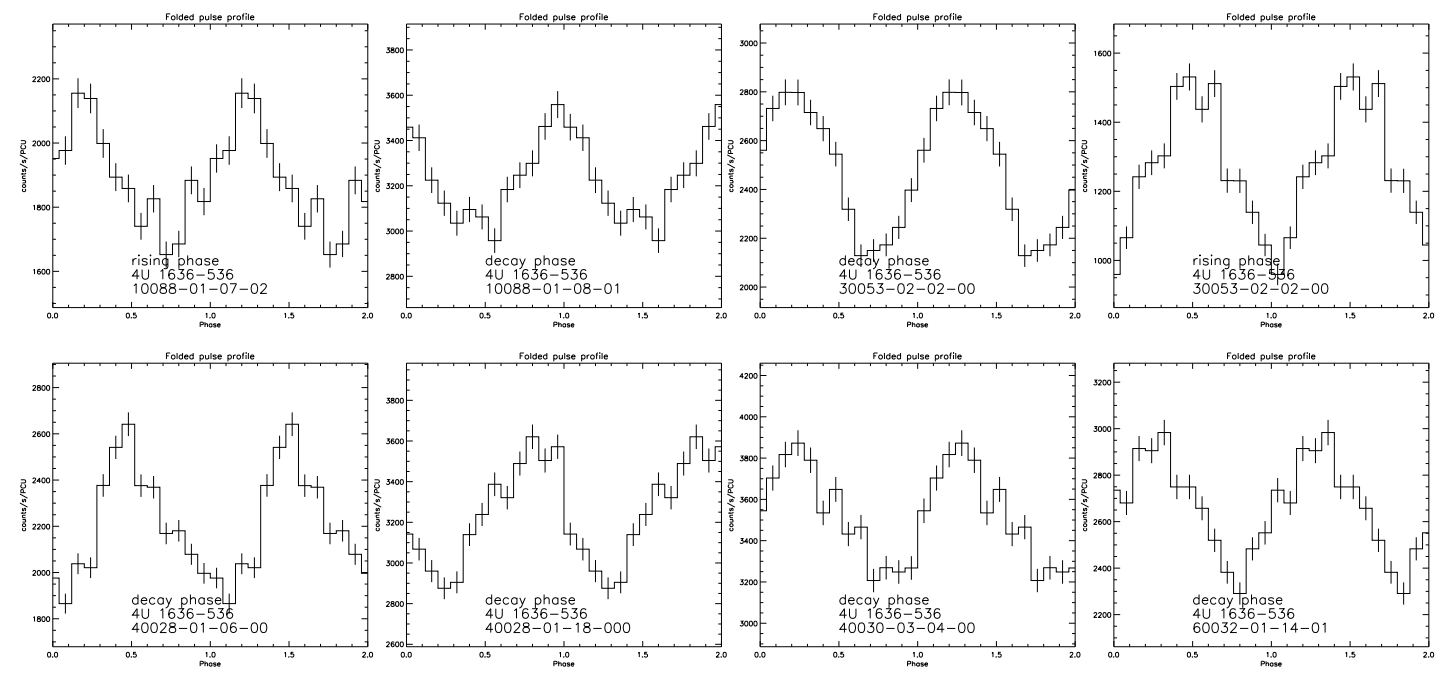

Figure 4: Folded pulse profiles for 4U 1636-536 bursts.

\section{Beyond the RXTE/PCA statistics}

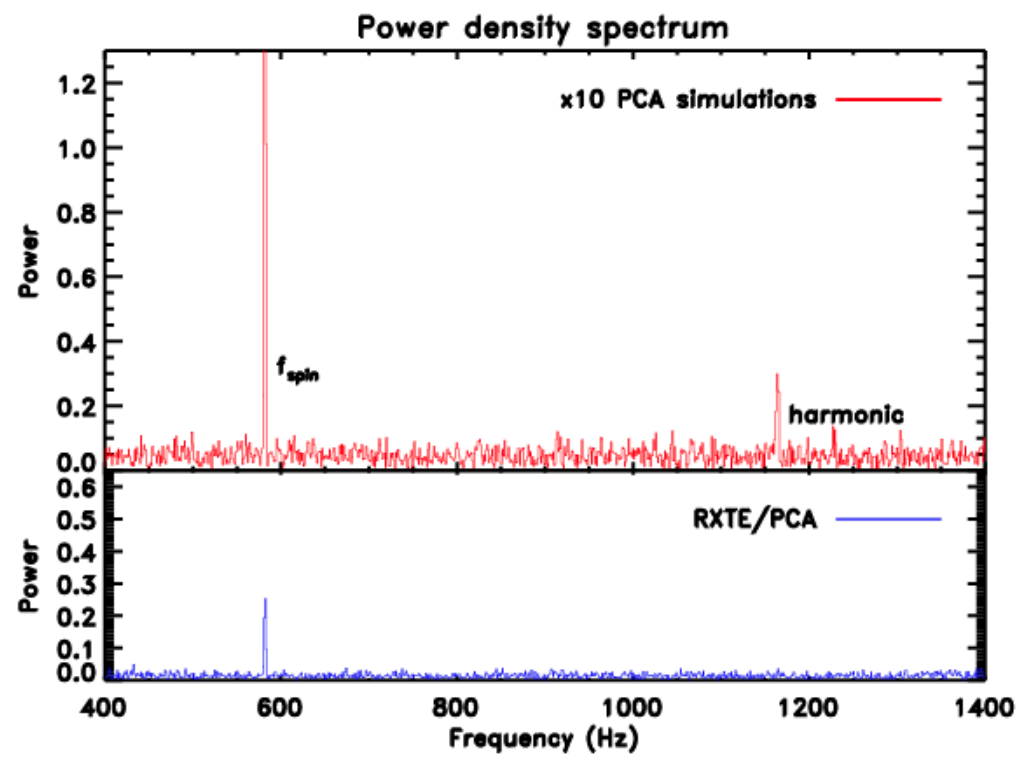

Figure 5: Power density spectra of burst light curve simulation with both the statistics of the PCA and an extreme count rate mission (10x PCA). The harmonic content become obvious in the latter case, while it was undetectable with the PCA.

As discussed above, the pulse profile is expected to be asymmetric. No such asymmetry is detected today with the PCA (and it is just that asymmetry that enables constraints on M and R to be derived). For illustrative purpose, we have simulated light curves with ten times better statistics than achieved by the PCA today. The Fast Fourier Transform of the X-ray simulated light curves (for a typical burst spectrum, blackbody of $2.5 \mathrm{keV}$ and a peak count rate of $13000 \mathrm{c} / \mathrm{s} / \mathrm{PCU}$ ) is shown in Figure 5, revealing the harmonic content of the signal. 


\section{Reverse engineering to constrain $M$ and $R$}

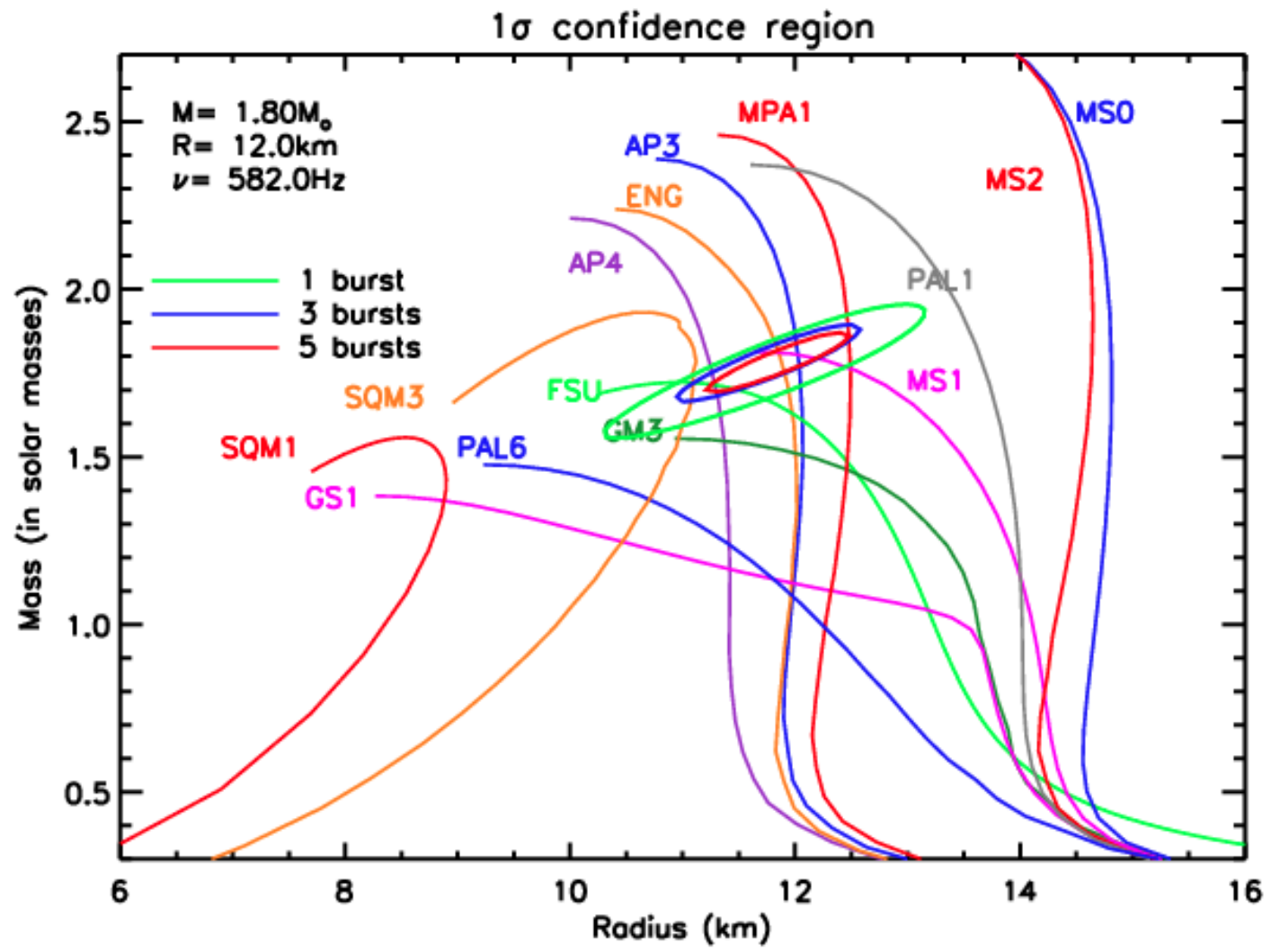

Figure 6: $1 \sigma$ confidence levels in the M-R diagram with extreme count rate data (10x PCA). We compute them for 1 single burst, but also for 3 then 5 co-added burst signals.

Next, we wished to check whether we could recover the simulated parameters from fitting of the folded pulse profiles by our library of theoretical light curves. For this purpose, we use a $\chi^{2}$ minimization procedure, allowing $\mathrm{M}$ and $\mathrm{R}$ to vary and assuming fixed hot spot geometry and emission pattern. $1 \sigma$ error contours are computed on $\mathrm{M}$ and $\mathrm{R}$. An example of such analysis is shown in Figure 6, showing that from one single burst, $\mathrm{M}$ and $\mathrm{R}$ can be constrained to $15 \%$ of their best fitted value. Improving further the statistics by co-adding bursts enables to reduce the ellipse region.

Repeating the same exercise with folded profiles with the PCA statistics would yield unclosed ellipses encompassing all the M-R curves.

\section{Conclusions}

We have shown that pulse profile fitting of X-ray bursts oscillations can lead to meaningful constraints on the mass and radius of neutron stars, provided that the statistical quality of the data can be improved by at least a factor of 10 compared to the data recorded today by the PCA. Such an improvement will be provided by LOFT (x20 PCA).

Allowing several bursts to be combined, we have shown that the statistical uncertainty on $\mathrm{M}$ 
and $\mathrm{R}$ can be reduced to less than $10 \%$, as required to discriminate between the various equations of state of dense matter.

A more complete work on both rising and decaying phase oscillations has to be done to better assess the information available in the RXTE archive, to include all the systematics uncertainties in the simulations (e.g. changing spot parameters from one burst to the other), and finally on the modeling of the burst emission (including broad-band spectroscopic study) and flame propagation.

\section{References}

[1] Lindblom, Lee, 1992, ApJ, 398, 569-573.

[2] Strohmayer, Tod E., 2004, Kaaret, P., Lamb, F.K., \& Swank, J.H., eds, AIP Conf. Proc., Vol. 714, X-Ray Timing 2003: Rossi and Beyond. Melville, NY, p.245.

[3] Cumming, Andrew, 2004, Nuclear Physics B (Proc. Suppl.) 132, 435-445

[4] Strohmayer, T. E. , Zhang, W., \& Swank, J.H., 1997, ApJ, 487 , L77ĐL80

[5] Poutanen, J. \& Beloborodov, A.M., 2006, MNRAS, 373, 836-844.

[6] Miller, M.C., \& Lamb, F.K., 1998, ApJ, 499, L37-L40

[7] In't Zand, Jean, http://www.sron.nl/\%7Ejeanz/bursterlist.html

[8] Nath, N.R., Strohmayer, T.E., \& Swank, J.H., 2002, Bounds on Compactness for Low-Mass X-Ray Binary Neutron Stars from X-Ray Burst Oscillations, ApJ, 564, 353-360 